\title{
DIVISION I / WORKING GROUP NUMERICAL STANDARDS OF FUNDAMENTAL ASTRONOMY
}

\author{
CHAIR \\ MEMBERS
}

\author{
Brian J. Luzum \\ Nicole Capitaine, Agnès Fienga, \\ William M. Folkner, Toshio Fukushima, \\ James L. Hilton, Catherine Y. Hohenkerk, \\ George A. Krasinsky, Gérard Petit, \\ Elena V. Pitjeva, Michael H. Soffel, \\ Patrick T. Wallace
}

\section{TRIENNIAL REPORT 2006 - 2009}

\section{Introduction}

The IAU Working Group (WG) on Numerical Standards for Fundamental Astronomy has been tasked with updating the IAU Current Best Estimates (CBEs), conforming with the IAU Resolutions, IERS Conventions and Système International d'Unités whenever possible. As part of its effort to achieve this, the WG is working in close cooperation with IAU Commissions 4 and 52, the IERS, and the BIPM Consultative Committee for Units.

This is the third IAU WG to be tasked with producing CBEs and is adding to the legacy of the two previous WGs. The first Sub-group on Numerical Standards of the IAU WG on Astronomical Standards was headed by E. M. Standish and the WG report (Standish 1995) established the rules which are still used today. For instance, this group decided on the two-tiered approach to the astronomical constants that we are currently using and also created the first CBEs for a list of IAU constants.

This work was continued by T. Fukushima and his IAU WG on Astronomical Standards (Fukushima 2000; Fukushima 2003). Many of the updates concerned work on constants in a general relativistic framework and improved estimates of the precession constant. This revised list of CBEs is the current IAU CBEs.

The excellent work of both these WGs has helped to establish the precedent and allows us to improve incrementally the values for which there are now better estimates.

\section{Changes since the last Current Best Estimates}

In addition to the need to update the CBEs because of improved estimates, there have also been significant changes that impact the IAU CBEs. Since the IAU CBEs were adopted, the IERS Conventions 2003, a document widely used by the astronomical and geodetic communities, has been produced. This reference contains estimates of many of the constants included in the IAU CBEs.

One significant change was the adoption of a new precession model with IAU 2006 Resolution B1. This resolution accepted the conclusion of the IAU Division I Working 
Group on Precession and the Ecliptic (Hilton et al. 2006) and adopted the P03 precession theory of Capitaine et al. (2003). This resolution also replaced the terms 'lunisolar precession' and 'planetary precession' with 'precession of the equator' and 'precession of the ecliptic'. Another change is the redefinition of Barycentric Dynamical Time (TDB) that occurred with the adoption of IAU 2006 Resolution B3.

These resolutions fundamentally alter the status of the associated constants. For instance, the general precession found in the IAU CBEs is no longer the appropriate quantity to describe precession. It should be replaced with either the rate of precession of the equator in longitude (or of the Celestial Intermediate Pole), or a number of precession quantities or expressions. The resolutions also changed the status of the constant $L_{B}$ to a defining constant.

\section{Changes to the Current Best Estimates}

The current WG started where the previous IAU WG tasked with providing CBEs left off, by using the existing IAU CBEs as the starting draft. From this starting point, the WG has proceeded to update the CBEs based on internationally adopted values and recent research. Some examples are adopting values:

- from the Committee on Data for Science and Technology (CODATA) 2006;

- recommended by the IAU 2006 Resolutions;

- based on recent research to modify most of the celestial object masses and adding masses for Ceres, Pallas, Vesta, and Eris. These improvements are possible due to years of high-precision observations of spacecraft as they near planets and their satellites.

The changes to the CBEs serve two significant purposes. First, they keep the IAU CBEs consistent, where possible, with international standards. They also keep the constants consistent with the most accurate estimates currently available.

To date, nine additional constants have been added to the list of CBEs, one constant has been superseded by another, one constant has been removed from the list and the numerical values for nine additional constants have been replaced by more current values. The draft version of the CBEs is available at <maia.usno.navy.mil/NSFA/CBE.html>.

\section{Additional concerns}

In addition to updating the list of CBEs, the WG is addressing the larger issues surrounding the adoption of IAU CBEs. These include the mechanism to keep the CBEs current and the way in which these constants will be provided, the procedure to document the theoretical context of the constants, and whether the IAU should revise its current list of adopted constants to correspond with the new list of CBEs.

The mechanism for maintaining the IAU CBEs has been discussed and, to date, three options have been considered. The current method is for the IAU to form a WG when it believes that the CBEs need to be updated. This method has worked in the past and there is no reason to believe that it would not work in the future. Another possibility would be to enlist the aid of the IERS Conventions Center to maintain the IAU CBEs. Since the IERS is an IAU service organization and it already has a mechanism in place to maintain CBEs, it is possible that the IERS Conventions could be used to maintain the IAU CBEs as well. In addition to providing a tested process of maintaining CBEs, this would also ensure that the IAU and IERS use consistent CBEs. The biggest problem with this method is that the user communities and the areas of research of the IERS and the IAU are slightly different and there is a possibility that these differences could 
be problematic. A third option is to create a permanent WG within IAU Commission 4 that would maintain the list of CBEs. One potential problem with this option is that in the past, the IAU has been reluctant to allow WGs to exist indefinitely. More details will need to be obtained to determine the potential status of a WG either within an IAU Commission or Division.

The numerical values for the CBEs are not numbers that exist in isolation; they are defined fully within the theoretical context in which they are estimated. The WG will need to account for this in the presentation of the CBEs by making the theoretical underpinnings apparent to the users of the CBEs. The level to which this is done and the method of achieving this are still under consideration.

Electronic information is likely to play an important role in achieving the proper level of documentation for the CBEs. The extent to which electronic information is used is also a topic that is still under consideration. Electronic media can be used as either a primary source for defining information or a secondary source, providing supplemental information.

Considering the possibility that a new IAU System of Constants will be proposed, there has been a brief discussion of the level of accuracy necessary for this system. In order to provide an appropriate response, it is necessary to understand the users and appreciate the uses of the system of constants. There has been preliminary discussion within the Working Group regarding the possibility of proposing both a list of CBEs for the users with the highest accuracy needs and a new IAU System of Constants with a reduced accuracy for a larger community of users. This issue is still under discussion.

\section{Update of IAU system of constants}

There are now significant differences between the CBEs and the current IAU system of constants. This is due to both increasing accuracy of estimates and to changes in astronomical theory. As a result of this, there is a consensus to recommend to the 2009 IAU General Assembly that the IAU System of Constants be updated. There is also a consensus to recommend that the IAU should seek a mechanism to periodically update the value of the astronomical constants and CBEs in the future.

$$
\begin{array}{r}
\text { Brian J. Luzum } \\
\text { chair of the Working Group }
\end{array}
$$

\section{References}

Capitaine, N., Wallace, P. T., \& Chapront, J. 2003, A\&\&A, 412, 567

Fukushima, T. 2000, in: K. J. Johnston, D. D. McCarthy, B. J. Luzum, \& G. Kaplan, (eds.) Proc. IAU Colloquium 180, Washington, DC, USA 27-31 March 2000, 417

Fukushima, T. 2003, in: H. Rickman (ed.), Highlights of Astronomy, 13, 107

Hilton, J. L., Capitaine, N., Chapront, J., Ferrandiz, J. M., Fienga, A., Fukushima, T., Getino, J., Mathews, P., Simon, J.-L., Soffel, M., Vondrak, J., Wallace, P., \& Williams, J. 2006, Celest. Mech. Dyn. Astr., 94, 351

Standish, E. M. 1995, in I. Appenzeller (ed.), Highlights of Astronomy, 12, 180 